\title{
Tuberculosis y actinomicosis. Un caso pediátrico de comorbilidad
}

\section{Pulmonary actinomycosis and tuberculosis. A comorbidity pediatric case}

\author{
Dra. Elsa D. Bisero ${ }^{a}$, Dra. Graciela F. Luque ${ }^{a}$ Dra. Cristina N. Rizzo ${ }^{a}$ Dra. Alejandra E. Zapata y \\ Dra. María S. Cuello
}

\begin{abstract}
RESUMEN
La actinomicosis es una infección supurativa crónica, producida porbacterias Gram-positivas anaeróbicas oespecies Actinomyces microaerófilas.

Es rara en niños y adolescentes; es más común en inmunodeprimidos. El Mycobacterium tuberculosis colabora en el desarrollo de la enfermedad.

La afectación pulmonaraparece como un cuadrode condensación crónica quenomejora con el tratamiento antibiótico convencional. Las complicaciones clásicas de afectación de la pared torácica con fistulización y supuración en «gránulo de azufre» son descritas con menor frecuencia en la actualidad.

El diagnóstico es un verdadero desafío y se establece mediante el aislamiento de las especies de Actinomyces. El tratamiento de elección para todas las formas clínicas de la enfermedad es el uso prolongado de antibióticos.

Objetivo. Presentar un caso pediátrico de comorbilidad entre tuberculosis y actinomicosis. Resaltar la importancia de la sospecha diagnóstica de actinomicosis frente a todo proceso supurado crónico.

Palabras clave: Mycobacterium tuberculosis, actinomicosis torácica, gránulos de azufre, Actinomyces spp., granuloma.
\end{abstract}

\begin{abstract}
Actinomycosis is a chronic suppurative infection, produced by anaerobic Gram-positive bacteria or microaerobic Actinomyces species. It is rare in children and adolescents and it is more common in immunocompromised. Mycobacterium tuberculosis collaborates on the development of the disease. Pulmonary involvement appears as a picture of chronic condensation that does not improve with conventional antibiotic treatment. Classic complications affecting the thoracic wall with drainage in «sulfur granule» and fistulization are described less frequently nowadays. The diagnosis is a real challenge and it is set by using the isolation of species of Actinomyces. The treatment of choice for all clinical forms of the disease is the prolonged use of antibiotics.
\end{abstract}

a. Sección Neumonología Infantil. Servicio de Pediatría.

Departamento Materno Infantil.

b. Sección Bacteriología. Servicio de Laboratorio.

c. Servicio de Anatomía Patológica.

Hospital Nacional Prof. A. Posadas.

Correspondencia: Dra. Elsa D. Bisero, elsibisero@yahoo.com.ar

Financiamiento: Ninguno.

Conflicto de intereses: Ninguno que declarar.

Recibido: 11-9-2015

Aceptado: 11-1-2016
Objective: to present a pediatric case of comorbidity between tuberculosis and actinomycosis and to highlight the importance of diagnostic suspicion of actinomycosis in the presence of all chronic suppurative processes.

Key words: Mycobacterium tuberculosis, thoracic actinomycosis, sulfur granules, Actinomyces spp., granuloma.

http:/ /dx.doi.org/10.5546/aap.2016.e233

\section{INTRODUCCIÓN}

La actinomicosis es una enfermedad indolente de evolución muy lenta y progresiva, producida por bacterias del género Actinomyces, que son comensales habituales de la flora de la boca, del colon y de la vagina. ${ }^{1}$ Se caracteriza por ser una infección supurativa crónica con producción de "gránulos de azufre", que se evidencian en las fístulas externas. ${ }^{2}$

Las manifestaciones clínicas son muy variadas. La localización más común es la oral y cervicofacial (50\%); otras regiones afectadas con menor frecuencia son la torácica, la abdominopélvica y el sistema nervioso central. Clínicamente, por lo general, es indistinguible de la tuberculosis y no suele ser considerada en el diagnóstico diferencial de las enfermedades pulmonares pediátricas.

Después de haber agotado un importante número de pruebas complementarias, el diagnóstico se establece por el hallazgo de Actinomyces. ${ }^{2}$ Responde al tratamiento antibiótico y suele tener buena evolución.,

\section{OBJETIVO}

Presentar un caso pediátrico de comorbilidad entre tuberculosis y actinomicosis.

\section{CASO CLÍNICO}

Paciente de sexo femenino, de 13 años de edad. Consultó por astenia, adinamia, hiporexia y pérdida de peso $(12 \mathrm{~kg})$ de 9 meses de evolución. Presentó fiebre, sudoración nocturna y tos productiva en los 2 últimos meses. Amenorrea secundaria de 10 meses de evolución. 
Antecedentes familiares: padre alcohólico fallecido 9 meses antes por sepsis con compromiso pulmonar.

Al momento de su ingreso, presentaba fiebre, desnutrición (índice de masa corporal -IMC-: 15,8; puntaje Z: -2) y síndrome de impregnación. Se palpaba una masa duro-pétrea izquierda, adherida a planos profundos, dolorosa, a nivel de la $12^{\mathrm{a}}$ costilla. Polo de bazo, indurado y doloroso. Hipoventilación en la base pulmonar izquierda. Vacuna BCG (bacilo de Calmette-Guérin), con dos cicatrices.

Exámenes complementarios: glóbulos blancos: $15700 / \mathrm{mm}^{3}$; hemoglobina: $8,4 \mathrm{~g} / \mathrm{dl}$; hematocrito: $31 \%$. Función renal y hepatograma: normales. Serologías: virus de inmunodeficiencia humana (VIH), antígeno de superficie de hepatitis B (HBs Ag) y prueba serológica para sífilis (venereal disease research laboratory; VDRL, por sus siglas en inglés), negativas. Proteinograma: hipergammaglobulinemia policlonal. Hemocultivos, negativos.

Radiografía de tórax: imagen radiopaca en el campo medio e inferior izquierdo (Figura 1).

Tomografía de abdomen de alta resolución: se observó colección que desplazaba el bazo (Figura 2).

Ecografía abdominal: hígado heterogéneo. Bazo heterogéneo, con áreas hiperecogénicas. Adenopatías retroperitoneales.

Ecografía de tórax: se observó una formación heterogénea de $5 \times 3 \mathrm{~cm}$ en la parrilla costal izquierda, en forma de reloj de arena, que se

FIgURA 1. Radiografía de tórax. Imagen radiopaca de limites difusos en el campo medio e inferior izquierdo con signo de silueta, que borra el seno costo y cardiofrénico

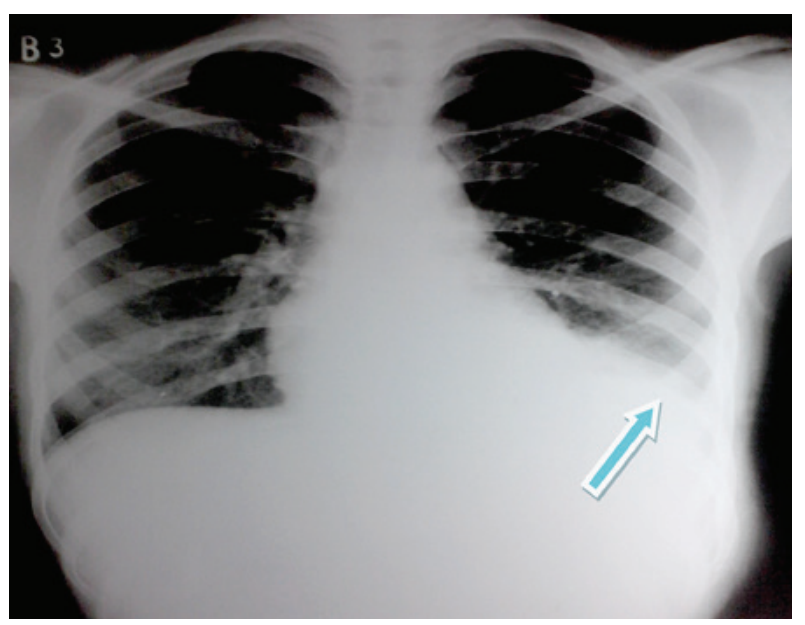

prolongaba a través del espacio intercostal, con centro abscedado (Figura 3).

Con diagnóstico de sepsis con absceso esplénico, se medicó con antibióticos y se decidió la conducta quirúrgica.

Al ingresar a la cavidad abdominal, se constató, en el espacio subfrénico izquierdo, gran colección $(1,5 \mathrm{~L})$ de material caseoso mezclado con material hemático, que se extendía hasta el hiato esofágico. Sobre la cara lateral del bazo, se vio una formación blanquecina, sangrante, redondeada. Impresionó salida de caseum. Pequeño bazo accesorio de $2 \mathrm{~cm}$ de diámetro. Por caída del hematocrito (24\%) y los hallazgos, se realizó una esplenectomía, por lo cual se inició profilaxis con penicilina.

Al $8^{\mathrm{vo}}$ día de internación, se interconsultó con Neumonología Infantil, quienes solicitaron

FIGURA 2. Tomografía axial computada de abdomen. Desplazamiento del bazo por una gran colección

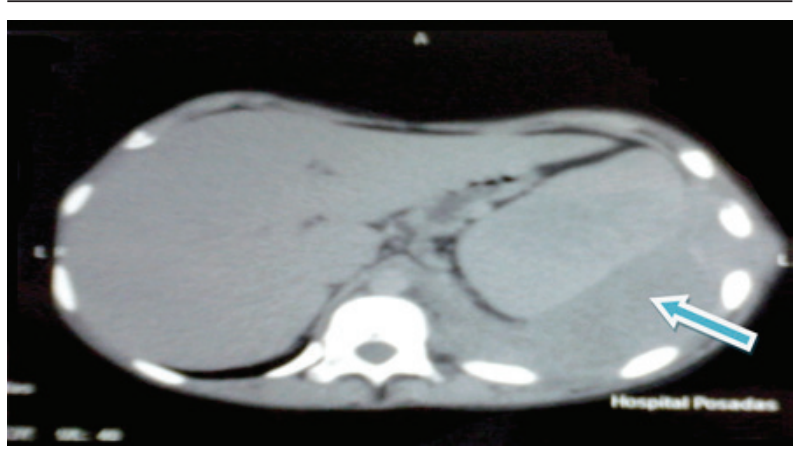

FIgURA 3. Ecografía torácica. Se observa una formación heterogénea de $5 \times 3 \mathrm{~cm}$ en la parrilla costal izquierda, en forma de reloj de arena, que se prolonga a través del espacio intercostal, con centro abscedado

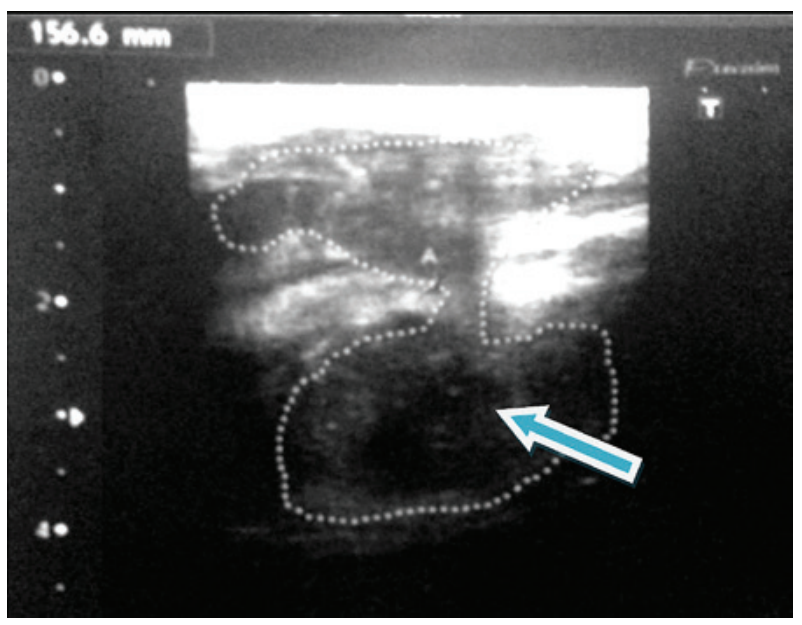


un examen microbiológico de esputo: tinción de Ziehl-Neelsen (ZN), negativa; cultivo, positivo (MGITTM 960).

Prueba tuberculínica (2 UT/0,1 ml de PPD RT23): $12 \mathrm{~mm}$.

Fondo de ojo: normal.

El estudio convencional de contactos fue negativo.

Con diagnóstico de tuberculosis diseminada de compromiso multiorgánico (pulmonar, peripleuritis, compromiso hepatoesplénico, ganglios retroperitoneales), se inició un tratamiento antituberculoso con $10 \mathrm{mg} / \mathrm{kg} /$ día de isoniacida $(\mathrm{H}), 15 \mathrm{mg} / \mathrm{kg} /$ día de rifampicina (R), $25 \mathrm{mg} / \mathrm{kg} /$ día de pirazinamida (Z) y $25 \mathrm{mg}$ / $\mathrm{kg} /$ día de etambutol (E) más vitamina $\mathrm{B}_{6}$.

A los 18 días, se recibió el cultivo de esputo: positivo para M. tuberculosis.

La lesión de partes blandas del tórax se tornó fluctuante, eritematosa, dolorosa; aumentó de tamaño y drenó espontáneamente. Impresionó gránulos de mijo. Se planteó la comorbilidad con actinomicosis.

A los 20 días de internación, se recibió el informe de anatomía patológica de bazo y secreción de partes blandas: parénquima esplénico alterado, sustituido por abscesos que rodeaban gránulos ovoides de color púrpura, que medían 100-300 $\mu \mathrm{m}$, rodeados por un infiltrado linfoplasmocitario, polimorfonucleares $\mathrm{y}$ abundantes histiocitos espumosos que se disponían en playas. Focos de fibrosis densa. ZN, negativa. Colonias bacterianas, positivas para Gram, ácido peryódico de Schiff (periodic acid

Figura 4. Anatomía patológica: histología. Microscopía: colonias de Actinomyces

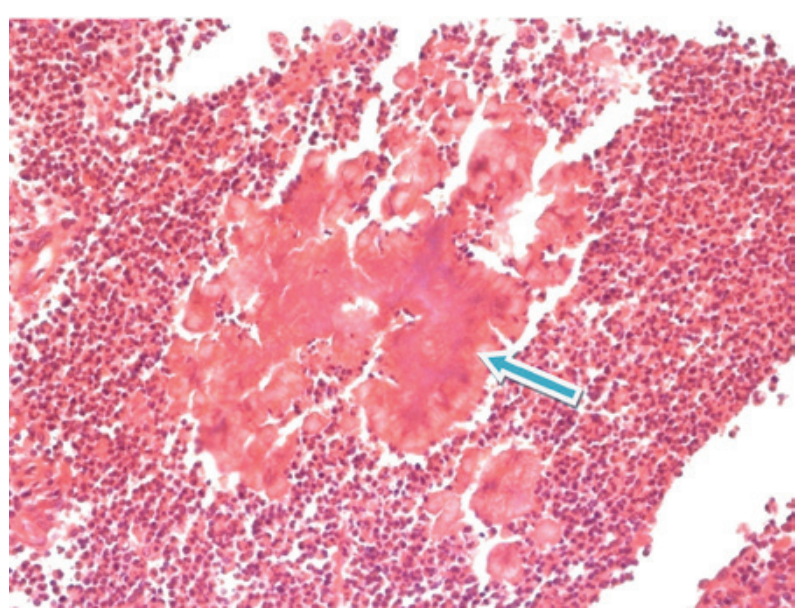

Schiff; PAS, por sus siglas en inglés) y Giemsa (Figura 4).

Se confirmó la asociación de tuberculosis y actinomicosis.

Evaluación odontológica y ginecológica, normal. Se indicaron las vacunas antineumocócica y meningocócica. Se dio el alta en buen estado general, con drogas antituberculosas y penicilina.

\section{COMENTARIO}

La actinomicosis humana fue descrita, en 1891, por M. Wolf y J. Israel. Es una infección causada por varias especies de Actinomyces, bacterias anaerobias facultativas, constituyentes de la flora normal de la orofaringe, del tracto gastrointestinal y genital femenino. El $A$. israelli es el más habitual en las infecciones humanas. ${ }^{5,6}$

La actinomicosis era frecuente en la era preantibiótica. Ha disminuido notablemente su incidencia. El 100\% de las personas están colonizadas a los dos años de edad. . $^{6} 7$

Es rara en pediatría; la mayoría se diagnóstica en las décadas medias de la vida.

A diferencia de nuestro caso, predomina en el género masculino. ${ }^{8-10}$

La desnutrición a cualquier edad constituye un factor desfavorable. Nuestra paciente estaba emaciada. ${ }^{11,12}$

Los sitios más comunes de presentación son las regiones cervicofacial (de $60 \%$ a $40 \%$ ), abdominopélvica (de $28 \%$ a $18 \%$ ) y torácica $(14 \%) .{ }^{13}$

Los Actinomyces causan infección cuando las barreras mucosas normales se alteran. Frecuentemente, lo hacen en conjunto con otros patógenos, que, por diversos mecanismos, colaboran en el desarrollo de la enfermedad. En el caso de nuestra paciente, existía la comorbilidad con la enfermedad tuberculosa.

La aspiración de secreciones nasofaríngeas es el fenómeno patogénico primario más aceptado para el desarrollo de actinomicosis torácica. Nuestra paciente tenía evaluación odontológica normal. ${ }^{8,14}$

Existen tres manifestaciones clínicas que deben hacer sospechar la enfermedad: a) Evolución crónica: progresión a través de fronteras tisulares y formación de masas. El caso presentado llevaba 9 meses de evolución y presentó una tumoración en la pared torácica. b) Desarrollo de un trayecto fistuloso, que puede resolverse espontáneamente y después reaparecer, tal cual ocurrió en nuestro caso. Y, c) Infección refractaria o recidivante luego de un ciclo de antibióticos. 
Los signos y síntomas inespecíficos tempranos semejan a los de la tuberculosis.

A nivel pulmonar, se presenta como una neumonía, empiema o masa endobronquial. La implicación de la costilla es frecuente. Los lóbulos pulmonares inferiores son más afectados, como en el caso expuesto.

En la evolución, presentó un empiema necessitatis (extensión de la supuración desde la pleura o parénquima pulmonar hacia la pared torácica). Este último cuadro puede también ser causado por enfermedades infecciosas, entre ellas, la tuberculosis, lo que da lugar al diagnóstico diferencial con epipleuritis tuberculosa.

En nuestra paciente, la biopsia de bazo y ganglio abdominal no arrojó granulomas tuberculosos.

No se identificó un claro foco de contagio. El papá había fallecido por una sepsis que incluía enfermedad pulmonar. No se pudo conocer el agente etiológico.

El diagnóstico de actinomicosis suele retrasarse por la dificultad en el cultivo, la falta de los característicos gránulos de azufre y el no ser considerado este agente etiológico entre los patógenos productores de enfermedad pulmonar en niños y jóvenes. En nuestro caso, el diagnóstico se sospechó con la fistulización y se confirmó con la anatomía patológica del bazo y la comorbilidad con tuberculosis por el esputo.

Los diagnósticos diferenciales incluyen sarcoma de Ewing, rabdomiosarcoma, neuroblastoma, tumor de Askin, linfoma, neurofibroma, linfangioma, leucemia.

En general, con una terapia apropiada, el pronóstico suele ser muy bueno, y se logra la curación en un alto porcentaje de los casos.

Se ha informado un rango de mortalidad del $0 \%$ al $28 \%$, que depende del sitio de la infección, el tiempo para el diagnóstico y el lapso transcurrido hasta iniciar el tratamiento. La evolución de nuestra paciente fue favorable. ${ }^{8}$

\section{CONCLUSIONES}

La comorbilidad entre actinomicosis y tuberculosis es poco frecuente en pediatría. Responde adecuadamente a dosis altas de penicilina, aun en casos graves. Se remarca la importancia del diagnóstico y del tratamiento temprano para evitar las secuelas.

\section{REFERENCIAS}

1. Cabrejos Perotti K, Pascual Gazquez JF, Alfayate Miguelez S, Trujillo Ascanio A. Actinomicosis: a propósito de 2 casos clínicos. An Pediatr (Barc) 2012;77(6):424-5.

2. Torres Ibérico Ro, Escalante E. Actinomicosis. Dermatol Peru 1999;9(1):45-7.

3. Watt AJ. Chest wall lesions. Paediatric Respir Rev 2002;3(4):328-38.

4. Choi J, Koh WJ, Kim TS, Lee KS, et al. Optimal duration of IV and oral antibiotics in the treatment of thoracic actinomycosis. Chest 2005;128(4):2211-7.

5. Sabbe LJ, Van De Merwe D, Schouls L, Bergmans A, Vaneechoutte $\mathrm{M}$, et al. Clinical spectrum of infections due to the newly described actinomyces species A. turicensis, A. radingae, and A. europaeus. JClin Microbiol 1999;37(1):8-13.

6. Mabeza GF, Macfarlane J. Pulmonary actinomycosis. Eur Respir J 2003;21(3):545-51.

7. Kapotsis GE, Daniil Z, Klimopoulos S, Malagari K, et al. A painful and swollen right breast in a young male. Eur Respir J 2004;24(6):1066-8.

8. Kwong JS, Müller NL, Godwin JD, Aberle D, et al. Thoracic actinomycosis: CT findings in eight patients. Radiology 1992;183(1):189-92.

9. Lee JP, Rudoy R. Pediatric thoracic actinomycosis. Hawaii Med J 2003;62(2):30-2.

10. Benammar S, Helardot PG, Sapin E, Adamsbaum C, et al. Childhood actinomycosis: report of two cases. Eur J Pediatr Surg 1995;5(3):180-3.

11. Chouabe S, Perdu D, Deslée G, Milosevic D, et al. Endobronchial actinomycosis associated with foreign body: four cases and a review of the literature. Chest 2002;121(6):2069-72.

12. Endo S, Murayama F, Yamaguchi T, Yamamoto S, et al. Surgical considerations for pulmonary actinomycosis. Ann Thorac Surg 2002;74(1):185-90.

13. Brook I. Actinomycosis. En: Goldman L, Schafer AI, eds. Cecil Medicine. 24th ed. Philadelphia: Saunders; 2011:1967-9.

14. Yeung VH, Wong QH, Chao NS, Leung MW, et al. Thoracic actinomycosis in an adolescent mimicking chest wall tumor or pulmonary tuberculosis Pediatr Surg Int 2008;24(6):751-4. 